14

\title{
Влияние механического нагружения на электрическую прочность полимеров
}

\author{
() А.И. Слуцкер ${ }^{1}$, Т.М. Велиев ${ }^{2}$, И.К. Алиева ${ }^{2}$, В.А. Алекперов ${ }^{2}$, Ю.И. Поликарпов ${ }^{3}$, Д.Д. Каров ${ }^{3}$ \\ ${ }^{1}$ Физико-технический институт им. А.Ф. Иофрфе РАН, \\ Санкт-Петербург, Россия \\ ${ }^{2}$ Институт фризики НАН Азербайджана, \\ Баку, Азербайджан \\ ${ }^{3}$ Санкт-Петербургский политехнический университет Петра Великого, \\ Санкт-Петербург, Россия \\ E-mail: Alexander.Slutsker@mail.ioffe.ru \\ (Поступила в Редакцию 23 июня 2016 г.)
}

\begin{abstract}
Зарегистрировано снижение электрической прочности (измеряемой как время ожидания пробоя диэлектрика в постоянном по знаку и величине электрическом поле) при растягивающем нагружении пленок полиэтилена и лавсана (полиэтилентерефталата) в области температур 100-300 К. Установлено, что вызываемое растягивающим нагружением полимера раздвижение осей соседствующих цепных молекул приводит к уменьшению энергетической глубины межмолекулярных ловушек электронов. Это ускоряет высвобождение электронов из ловушек посредством надбарьерного перехода при повышенных температурах $(\sim 230-350 \mathrm{~K})$ и туннельного перехода при пониженных температурах ( 80-200 K) в аморфных областях полимеров. В результате происходит сокращение времени формирования критического объемного заряда времени ожидания пробоя, что и означает снижение электрической прочности полимеров.
\end{abstract}

DOI: $10.21883 /$ FTT.2017.01.43972.258

\section{1. Введение}

Электрической прочностью диэлектриков принято называть устойчивость к пробою - образованию проводящего канала в слое диэлектрика, находящегося между электродами, при приложении электрического напряжения. При представлении пробоя как явления критического характера, наступающего при достижении прикладываемым напряжением определенной величины, за меру электрической прочности принималась величина напряженности (отношение электрического напряжения к толщине слоя диэлектрика), называемая пробивной напряженностью [1]. В данной работе рассматривается условие приложения только постоянного (по знаку) электрического напряжения.

Использование в качестве меры электрической прочности величины пробивной напряженности встречало сложности, поскольку такая мера оказалась неоднозначной. Так, величина пробивной напряженности для данного диэлектрика изменялась с температурой и зависела от скорости подъема прикладываемого напряжения до пробивного значения. Отмечалось также, что пробивная напряженность $E_{m}$ зависела от времени пребывания диэлектрика под действием электрического поля. Непостоянство пробивной напряженности диэлектрика плохо согласовывалось с пониманием пробоя как критического явления.

Объяснение было найдено тогда, когда стало ясно, что электрический пробой диэлектриков выступает не как событие критического характера, а как явление, имеющее кинетическую природу. Это обнаружилось в результате своеобразной „инверсии“: перехода от „привычной“ зависимости $E_{m}(\tau)$ к зависимости $\tau_{e}(E)$, в которой величина $\tau_{e}$ получила название электрической долговечности. Физический смысл зависимости $\tau_{e}(E)$ является весьма простым и наглядным: действие напряженности между электродами на диэлектрик вызывает в нем процесс, доводящий диэлектрик до пробоя. Интенсивность этого процесса зависит от величины напряженности, температуры и других возможных факторов воздействия. Многочисленные исследования были направлены на выяснение природы этого процесса (получившего название процесса электрического разрушения диэлектрика). Результаты такого исследования приведены далее.

Здесь же отметим, что появилась новая мера электрической прочности диэлектриков - электрическая долговечность $\tau_{e}$. Эта мера имеет определенность при задаваемых или известных значениях напряженности, температуры и других возможных факторов. При этом эксперимент показывает, что при определенных значениях $E, T$ и др. имеет место разброс значений $\tau_{e}$ (распределение по $\tau_{e}$ ). Тогда в качестве меры электрической прочности выступает среднее значение долговечности при фиксированных значениях $E, T$ и др. Электрическая прочность тем выше, чем продолжительнее электрическая долговечность.

Для полимерных диэлектриков обнаружено интересное явление: изменение электрической долговечности при механическом нагружении образцов [2,3], т.е. обнаружено воздействие механической нагрузки на электронную подсистему полимеров. Детального анализа зависимости $\tau_{e}(\sigma)(\sigma-$ механическое напряжение) с выяснением механизма данного „электромеханического“ 
эффекта фактически не проводилось. Задачей настоящей работы является такое выяснение.

\section{2. Объекты и метод исследования}

Объектами исследования были избраны простые всесторонне изученные линейные полимеры: полиэтилен (ПЭ) и полиэтилентерефталат (ПЭТФ), часто называемый лавсаном [4].

Образцы брались в виде неориентированных пленок толщиной $20-40 \mu \mathrm{m}$. Они заключались между плоскими металлическими прижимными электродами, на которые подавалось постоянное по знаку электрическое напряжение величиной до $10-20 \mathrm{kV}$. Образцы находились в зажимах для механического растягивающего нагружения. Все электронагружающее устройство помещалось в термостат, позволяющий устанавливать и поддерживать в образце температуру в диапазоне $100-300 \mathrm{~K}$. После установления требуемой температуры $T$ на образцы одновременно подавалось электрическое напряжение и прикладывалось одноосное растягивающее механическое напряжение величиной до $60 \mathrm{MPa}$. Механические напряжения выбирались небольшими, чтобы избежать значительной ориентационной вытяжки образцов полимеров. Ось механического растяжения и вектор электрической напряженности в образцах между электродами были ортогональными друг другу.

В условиях комбинации электрического и механического воздействия образец выдерживался до наступления пробоя и определялась величина электрической долговечности $\tau_{e}$.

Вследствие характерного разброса $\tau_{e}$ для каждой комбинации условий $(E, T$ и $\sigma)$ производились измерения $\tau_{e}$ для серии из 15-20 образцов, и находилось среднее значение $\lg \tau_{e}$.

\section{3. Результаты измерений}

На рис. 1 приведены измеренные исходные (без механического нагружения) температурные зависимости электрической долговечности пленок ПЭ $(a)$ и ПЭТФ $(b)$ в аррениусовских координатах $\lg \tau_{e}-T^{-1}$ при заданном значении электрической напряженности $E=0.5 \mathrm{GV} / \mathrm{m}$.

На рис. 2 показаны результаты приложения механического растягивающего нагружения при том же значении электрической напряженности для пленок ПЭ $(a)$ и ПЭТФ $(b)$. Из данного рисунка видно, что величина растягивающего напряжения $\sigma$ систематически уменьшает электрическую долговечность полимеров: ускоряет процесс подготовки полимера к пробою (снижает электрическую прочность).

\section{4. Анализ и обсуждение результатов}

Показанные на рис. 1 исходные (без механического нагружения) зависимости $\lg \tau_{e}\left(T^{-1}\right)$ имеют вид, характерный для диэлектриков различной структуры (керамик и
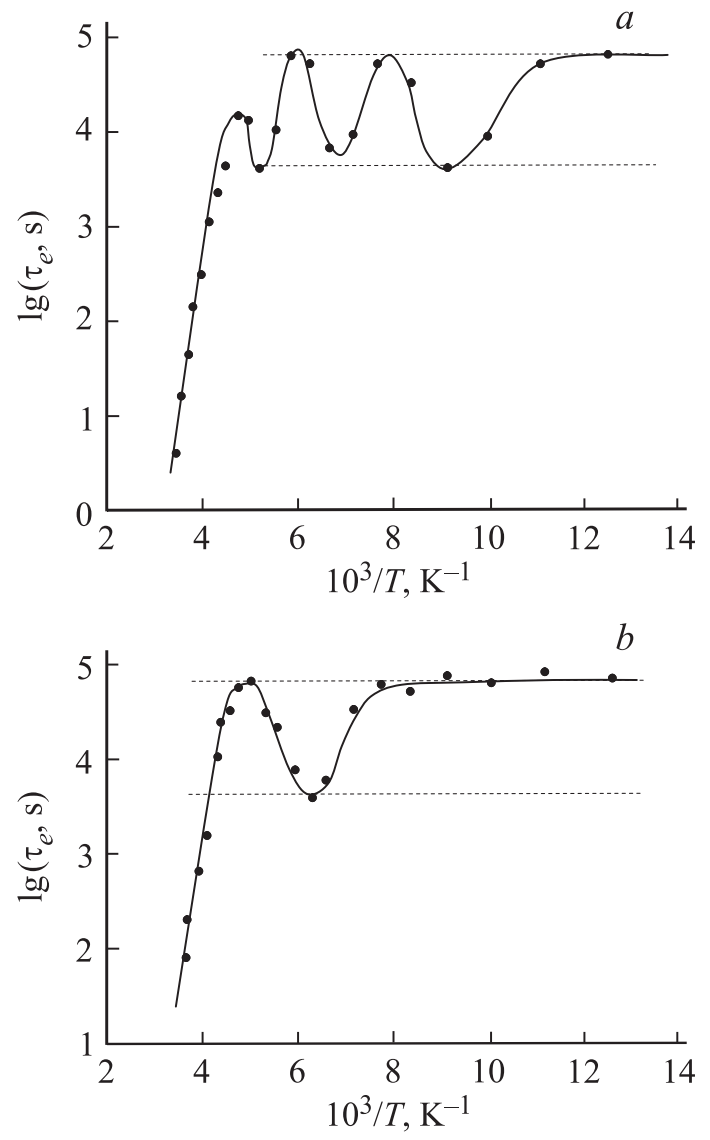

Рис. 1. Температурные зависимости электрической долговечности полимеров. $a-$ ПЭ, $b-$ ПЭТФ. Напряженность электрического поля $E=0.5 \mathrm{GV} / \mathrm{m}$.

полимеров): состоят из двух участков $[5,6]$. Для полимеров в области повышенной температуры $(\sim 220-300 \mathrm{~K})$ зависимости близки к линейным с „крутым“ наклоном и охватывают диапазон $\tau_{e}$ в три-четыре десятичных порядка. В области пониженных температур $(\sim 80-220 \mathrm{~K})$ зависимости лежат в сравнительно узких горизонтальных коридорах (высотой $\Delta \lg \tau_{e} \sim 1$ ). Верхние и нижние границы коридоров показаны штриховыми прямыми на рис. 1. В горизонтальных низкотемпературных коридорах (при атермичности оси коридора) наблюдаются температурные „волнообразные“ отклонения от верхней границы.

Анализ зависимостей $\lg \tau_{e}(E, T)$ для полимеров привел к выяснению механизма кинетики электрического разрушения полимеров $[5,6]$. Электрическое напряжение на металлических электродах вызывает инжекцию из электродов в полимер: электронов - из катода, дырок - из анода. Инжектированные заряженные частицы внутри полимера попадают в ловушки различной энергетической глубины, в том числе и глубокие $(\sim 2 \mathrm{eV})$. Приложенное электрическое поле снижает барьеры выхода заряженных частиц из ловушек. При повышенных температурах происходят термофлуктуационные надбарьерные выходы из глубоких ловушек с резкой экспоненциальной зависимостью их вероятности от темпера- 

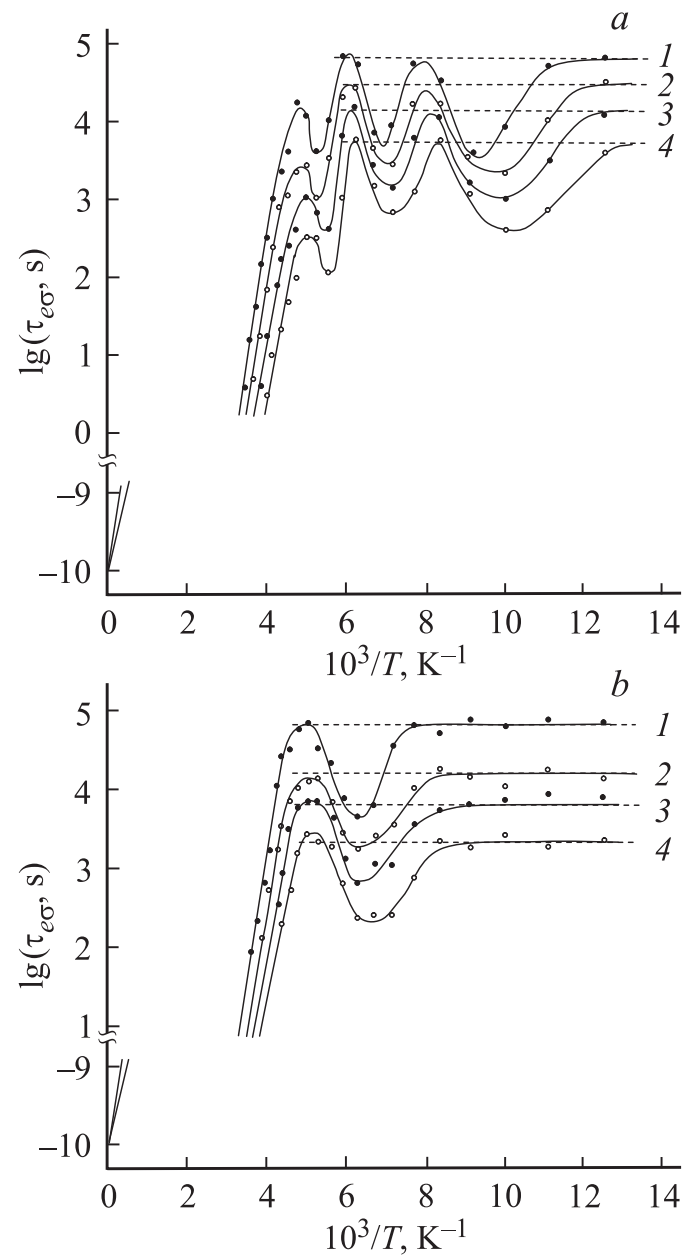

Рис. 2. Температурные зависимости электрической долговечности полимеров при действии механического растягивающего напряжения. $a-$ ПЭ, $b-$ ПЭТФ. Напряженность электрического поля $E=0.5 \mathrm{GV} / \mathrm{m}$. Напряжение механической нагрузки $\sigma=0$ (1), 20 (2), 40 (3), $60 \mathrm{MPa}(4)$.

туры. При низких температурах происходят туннельные подбарьерные выходы из глубоких ловушек атермического характера. В обоих случаях выходы из ловушек приводят к дрейфу зарядов различного знака навстречу друг другу, накоплению и сближению объемных зарядов разного знака и при достижении за достаточное время (электрическая долговечность!) критического значения величины объемных зарядов происходит рекомбинация зарядов, носящая „взрывной“ характер, что и вызывает пробой полимера.

„Волны“ в горизонтальных атермических коридорах (рис. 1) связаны с тем, что при некоторых температурах (в низкотемпературной области) в полимерах происходят релаксационные (конформационного типа [7]) переходы с невысокими энергиями активации $(0.3-0.5 \mathrm{eV})$, которые изменяют (несколько уменьшают) энергетическую глубину глубоких ловушек, оставляя выход из этих ловушек туннельным [6].

Таким образом, кинетика электрического разрушения представляет собой процесс, состоящий из элементар- ных актов - выходов заряженных частиц из ловушек, приводящий к накоплению критических объемных зарядов, вызывающих пробой полимерного образца.

Выясненный механизм электрического разрушения позволяет предположить объяснение влияния механического нагружения на процесс в электронной подсистеме полимеров.

Существенную роль в объяснении факта влияния „механики“ на электрический процесс в полимерах играет определяющее участие ловушек в механизме электрического разрушения. Более того, именно специфический вид глубоких ловушек в полимерах ведет к достаточно простому и наглядному варианту объяснения ускорения электрического разрушения (снижения электрической прочности) полимеров.

Глубокими ловушками в полимерах принято считать промежутки между цепными молекулами $[8,9]$. В этих промежутках располагаются боковые атомы - „привески“ к скелету полимерной молекулы, состоящему преимущественно из атомов углерода. „Привески“ же состоят главным образом из атомов водорода. Таким образом, водородные „Привески“ к соседствующим молекулам образуют некоторое подобие „клетки“ для оказавшегося в межмолекулярном промежутке электрона. Взаимодействие электрона с окружающими его водородными атомами и определяет энергетическую глубину ловушки и тем самым высоту потенциального барьера выхода электрона из ловушки. Представляется, что чем менее „тесно“ электрону в ловушке, тем слабее взаимодействие электрона со „стенками“ клетки-ловушки и тем меньше энергетическая глубина ловушки и соответственно меньше высота барьера выхода электрона из ловушки. Тогда на основе такого представления можно предположить механизм влияния механического нагружения полимера на величину его электрической долговечности.

Основу предположительного механизма составляет соображение о том, что растягивающее механическое напряжение может вызывать раздвижение соседних цепных молекул. Это приводит к увеличению ширины глубоких ловушек (расстояния между их „стенками“), уменьшающему энергетическую глубину ловушек, и, следовательно, к снижению высоты барьера выхода электрона из глубоких ловушек и тем самым вызывает уменьшение электрической долговечности полимера.

Теперь следует выяснить, имеются ли экспериментальные подтверждения названного „ловушечного“ механизма исследуемого электромеханического эффекта в полимерах.

В [6] для тех же полимерных объектов (пленок ПЭ и ПЭТФ) были обоснованы и применены приближенные выражения для описания электрической долговечности полимеров:

в области повышенных температур $\sim 230-350 \mathrm{~K}$ (кинетика классических, надбарьерных переходов)

$$
\tau_{e} \approx \tau_{a} \exp [U(E) / k T]
$$

где $U(E)$ - зависящий от напряженности поля барьер, $\tau_{a} \approx 10^{-10} \mathrm{~s}, T-$ температура, $k-$ постоянная Больцмана; 
в области пониженных температур $\sim 80-200 \mathrm{~K}$ (кинетика квантовых, туннельных переходов)

$$
\tau_{e} \approx \tau_{b} \exp \left\{\frac{8 \pi \varepsilon\left(2 m_{e}\right)^{1 / 2}[U(E)]^{3 / 2}}{3 e h E}\right\},
$$

где $\tau_{b} \approx 10^{-4}-10^{-5} \mathrm{~s}, e$ и $m_{e}$ - заряд и масса электрона, $\varepsilon-$ диэлектрическая проницаемость, $h-$ постоянная Планка.

Из рис. $1, a$ и $b$ или из рис. 2, $a$ и $b$ (кривые 1 ) с помощью выражений (1) и (2) получены значения высоты барьеров выхода электронов из ловушек при напряженности электрического поля $E=0.5 \mathrm{GV} / \mathrm{m}$ в полимерах еще до их механического нагружения (когда механическое напряжение $\sigma=0$ ).

При нахождении высоты барьера при низких температурах в выражении, следующем из (2), здесь и далее принимались значения $\lg \tau_{e}$, отвечающие верхним границам горизонтальных коридоров на рис. 1 и 2.

Значения $U(0.5 \mathrm{GV} / \mathrm{m})$, найденные при повышенных (по выражению (1)) и при пониженных (по выражению (2)) температурах, оказались близкими (соответственно 0.62 и $0.8 \mathrm{eV}$ для ПЭ и 0.63 и $0.65 \mathrm{eV}$ для ПЭТФ). Поскольку при повышенных и при пониженных температурах „работают“ одни и те же межмолекулярные ловушки, барьеры выхода из этих ловушек при разных температурах для данного полимера должны быть практически одинаковыми. Проявляющаяся разница в измеряемых значениях барьеров является следствием приближений и допущений при обработке результатов измерений. Поэтому далее будем использовать средние значения $U: 0.71 \mathrm{eV}$ для ПЭ и $0.64 \mathrm{eV}$ для ПЭТФ при $\sigma=0$.

Перейдем к рассмотрению влияния механического нагружения на электрическую долговечность полимеров.

Полагаем, что регистрируемое по данным рис. 2, $a$ и $b$ уменьшение долговечности с ростом механического растягивающего напряжения $\sigma$ является следствием уменьшения высоты барьера $U$. Свидетельством этого может служить вид выражений (1) и (2), в которых изменение $\lg \tau_{e}$ фактически связано только с одной способной изменяться величиной - барьером $U$ (напряженность поля $E$ и температура $T$ здесь задаются и поддерживаются постоянными, а предэкспоненты в (1) и (2) в логарифмическом масштабе могут считаться постоянными).

Таким образом, можно принять, что зависимость $\lg \tau_{e}(\sigma)$ обусловлена зависимостью $U(\sigma)$.

В этом случае применялись выражения (1) и (2), в которых вместо величины $U(E)$ использовалась $U(\sigma)$, и по измеряемым значениям $\lg \tau_{e}(\sigma)$ на рис. 2 были найдены зависимости $U(\sigma)$ и для ПЭ, и для ПЭТФ. Значения $U(\sigma)$ для повышенных и низких температур в обоих полимерах и здесь оказались близкими (разница $\sim 30 \%$ для ПЭ и $\sim 15 \%$ для ПЭТФ). Поэтому для зависимостей $U(\sigma)$ опять используем средние (между полученными для повышенных и низких температурах)

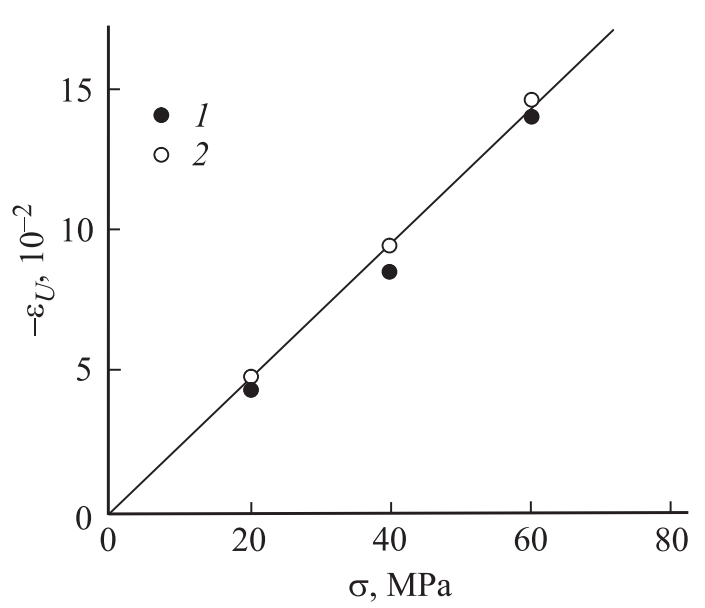

Рис. 3. Снижение барьера выхода электрона из ловушки действием растягивающей нагрузки. $1-$ ПЭ, $2-$ ПЭТФ.

значения высоты барьера $U(\sigma)$ для каждого из полимеров (ПЭ и ПЭТФ).

Найденные зависимости относительного изменения (уменьшения) высоты барьера с нагрузкой

$$
\varepsilon_{U}(\sigma)=\frac{\Delta U(\sigma)}{U(\sigma=0)}=\frac{U(\sigma)-U(\sigma=0)}{U(\sigma=0)}
$$

приведены на рис. 3 для ПЭ и ПТФЭ. Эти зависимости (оказавшиеся близкими для ПЭ и ПЭТФ, что, очевидно, является случайностью, связанной, как уже отмечалось, со многими приближениями при определении $U(\sigma))$, аппроксимируемые одной общей линией, демонстрируют практически прямую пропорциональность уменьшения барьера выхода электронов из ловушек величине прикладываемой механической растягивающей нагрузки.

Исходя из предложенного механизма действия растягивающего механического напряжения $\sigma$ на электрическую долговечность полимеров зависимость $\varepsilon_{U}(\sigma)$ следует понимать как зависимость барьера от ширины ловушки, т.е. $\varepsilon_{U}(\sigma)=\varepsilon_{U}\left[\varepsilon_{\mathrm{tr}}(\sigma)\right]=\varepsilon_{U}\left(\varepsilon_{\mathrm{tr}}\right)$, где $\varepsilon_{\mathrm{tr}}(\sigma)-$ относительное увеличение ширины межмолекулярной ловушки, вызываемое растягивающим напряжением.

Проведем экспериментальную оценку зависимости увеличения ширины межмолекулярных ловушек от растягивающего напряжения. Ширину такой ловушки примем равной расстоянию между осями соседних цепных полимерных молекул $l_{\perp}$. Для этой оценки используем результаты измерения растяжения пленок ПЭ и ПЭТФ приложенным механическим напряжением $(\sigma=20,40$, $60 \mathrm{MPa})$. Результаты измерения относительного растяжения $\varepsilon_{L}=\Delta L(\sigma) / L_{0}\left(L_{0}-\right.$ исходная длина растягиваемой полимерной пленки) показаны на рис. 4.

Видно, что зависимости $\varepsilon_{L}(\sigma)$ линейны и близки к прямопропорциональным.

Поскольку линейные зависимости $\varepsilon_{L}(\sigma)$ для ПЭ и ПЭТФ несильно отличаются друг от друга (разница $\sim 20 \%$ ), для дальнейших сравнений примем усредненную между ПЭ и ПЭТФ зависимость $\varepsilon_{L}(\sigma)$, показанную линией 3 на рис. 4. 


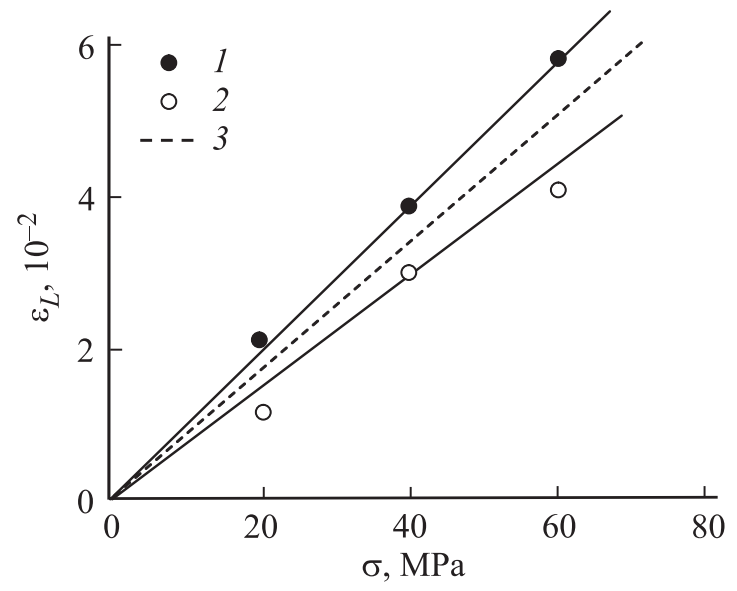

Рис. 4. Растяжение полимеров действующей нагрузкой. 1 ПЭ, 2 - ПЭТФ, 3 - средняя между 1 и 2 зависимость.

Эксперименты показали, что $\varepsilon_{L}(\sigma)$ слабо зависят от температуры в диапазоне 300-77 К и, что важно, деформации являются практически обратимыми (упругими). Подчеркнем, что зависимости $\varepsilon_{L}(\sigma)$ относятся к измеренным макроскопическим деформациям пленок, которые представляют собой усреднения по объему полимера локальных микроскопических деформаций элементов структуры полимеров с вариациями их упругости, размеров и молекулярной ориентации. По смыслу решаемой задачи требуется значение не макроскопической деформации, а локальной деформации - изменения межмолекулярного расстояния в элементах структуры полимеров.

Исследуемые полимеры (ПЭ и ПЭТФ) по структуре являются аморфно-кристаллическими [10]. Объем этих полимеров состоит из кристаллитов и межкристаллитных аморфных участков. Жесткость этих элементов структуры резко различается: жесткость кристаллитов значительно превышает жесткость аморфных участков [11]. Поэтому возникающая при нагружении деформация полимерного образца в значительной (вплоть до подавляющей) степени является деформацией аморфной части объема полимера. Характерная степень кристалличности (доля объема полимера, включающая кристаллиты) таких полимеров, как ПЭ и ПЭТФ, составляет $\eta_{\text {cr }} \sim 0.5-0.6$ [10]. Тогда, учитывая доминирующую роль деформации аморфной части объема полимера и пренебрегая вкладом деформации кристаллической части, приходим к соотношению между деформацией аморфной части $\varepsilon_{\text {am }}$ и полной (макроскопической) деформацией полимерного образца $\varepsilon_{L}(\sigma)$

$$
\varepsilon_{\mathrm{am}}(\sigma) \approx \varepsilon_{L}(\sigma) /\left(1-\eta_{\mathrm{cr}}\right) \approx 2 \varepsilon_{L}(\sigma)
$$

Но кроме „кристаллитного“ вклада следует учесть и еще один фактор - вариацию локальной ориентации элементов аморфной структуры полимеров. Подчеркнем, что результаты действия механической нагрузки на элементы молекулярно-цепной структуры полимеров отличаются резкой анизотропией. В каждом элементе структуры полимера, состоящего из небольшого числа контактирующих участков макромолекул, имеются преимущественные направления осей цепных молекул. В этом направлении жесткость участка структуры при действии одноосной нагрузки является сравнительно высокой ввиду большой величины модуля продольной упругости скелета молекул, построенного на сильных ковалентных связях между скелетными атомами. В поперечных же относительно осей молекул направлениях действуют значительно более слабые межмолекулярные связи (ван-дер-ваальсовы либо водородные), что и определяет низкий модуль упругости участка структуры в поперечном направлении. В результате в тех местах аморфной части объема полимера, где приложенное механическое напряжение действует вдоль осей молекул в участке структуры, деформация участка незначительна, а в местах ортогонального направления нагрузки к осям молекул деформация участка будет существенно больше. В результате такие участки будут иметь преимущественно поперечную деформацию. Это означает, что при растягивающем нагружении в этом участке оси составляющих его молекул будут несколько раздвигаться: возрастает ширина глубоких межмолекулярных ловушек. Исследованные полимеры находились в неориентированном состоянии, в котором оси участков структуры имели хаотическую ориентацию. Тогда исходя из статистики можно принять, что доля аморфных участков, оси молекул в которых близки к ортогональному относительно оси растягивающей силы направлению, составляет $\sim 2 / 3$. Деформация именно этих участков доминирующим образом определяет деформацию всей аморфной части объема полимера. Тогда относительное раздвижение осей молекул в этих участках при учете (3) составит

$$
\varepsilon_{\mathrm{mol}}(\sigma)=3 \varepsilon_{\mathrm{am}}(\sigma) / 2 \approx 3 \varepsilon_{L}(\sigma) .
$$

Таким образом, из измеренной зависимости растяжения полимерных пленок $\varepsilon_{L}(\sigma)$ получаем оценочное значение увеличения ширины глубоких межмолекулярных ловушек

$$
\varepsilon_{\mathrm{tr}}(\sigma)=\varepsilon_{\mathrm{mol}}(\sigma) \approx 3 \varepsilon_{L}(\sigma) .
$$

После этого линейную зависимость уменьшения барьера выхода электрона из ловушки от напряжения $\varepsilon_{L}(\sigma)$ (рис. 3) (практически одинаковую для ПЭ и ПЭТФ) перестраиваем, пользуясь усредненной для ПЭ и ПЭТФ зависимостью $\varepsilon_{L}(\sigma)$, в зависимость изменения высоты барьера от увеличения ширины ловушки $\varepsilon_{U}\left(\varepsilon_{\mathrm{tr}}\right)$, приведенную на рис. 5.

Видно, что линейная зависимость $\varepsilon_{U}\left(\varepsilon_{\mathrm{tr}}\right)$ (общая для обоих полимеров) оказалась близкой к биссектрисе координатного угла, показанной штриховой прямой на pис. 5. Это означает, что при каждой нагрузке $\sigma$ относительные значения $\varepsilon_{U}$ и $\varepsilon_{\mathrm{tr}}$ получаются близкими

$$
-\varepsilon_{U}(\sigma) \approx \varepsilon_{\mathrm{tr}}(\sigma) .
$$

Возникает вопрос: имеет ли такая близость относительных изменений высоты барьера и ширины ловушки $\left(\varepsilon_{U}\right.$ 


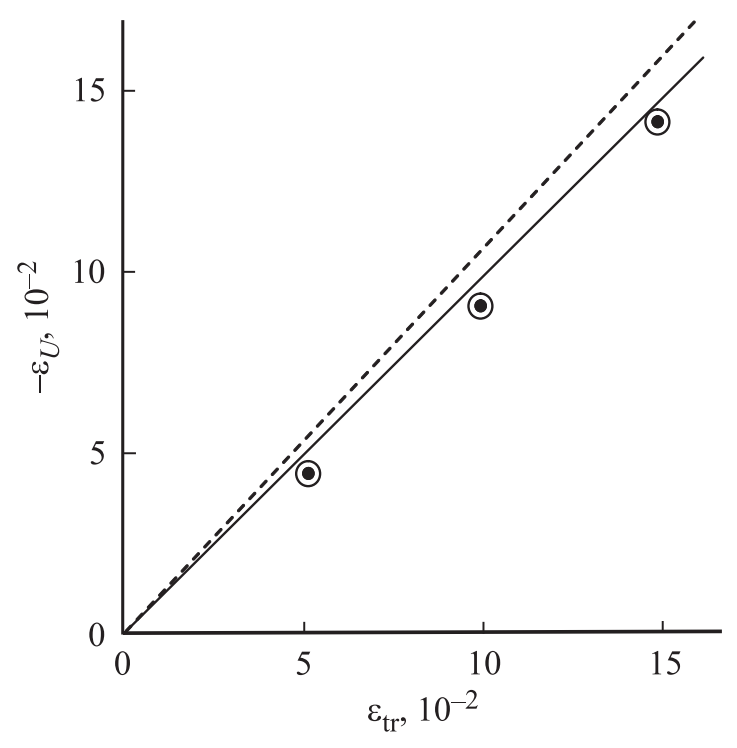

Рис. 5. Связь снижения барьера выхода электрона с увеличением ширины ловушки при растягивающем нагружении полимеров. Штриховая линия - биссектриса координатного угла.

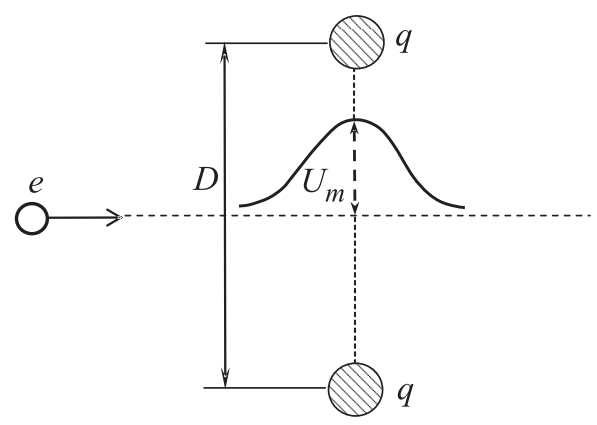

Pис. 6. Схема потенциального барьера при прохождении заряженной частицы $(e)$ между одноименно заряженными частицами $(q)$.

и $\varepsilon_{\text {tr }}$ ) (получившаяся высокая степень которой, конечно, случайна) физические (расчетно-теоретические) обоснования?

Поскольку взаимодействие заряженной частицы в ловушке с атомами межмолекулярной „клетки“ является весьма сложным, уже результаты расчетов энергетической глубины ловушек носят приближенный характер, и полученные значения глубоких межмолекулярных ловушек $(1.5-2.0 \mathrm{eV}[9,12])$ являются оценочными. Тем более пока вряд ли возможно сделать сколько-нибудь точным расчет изменения энергетической глубины ловушки при изменении взаиморасположения „стенок“ ямы-ловушки. Поэтому представляется полезным привлечь для обсуждения изменения высоты барьера выхода заряженной частицы из ловушки крайне упрощенную модель прохождения заряженной частицы (заряд $e$ ) между двумя заряженными частицами того же знака (заряды q) (рис. 6). Принимая взаимодействие между заряженными частицами кулоновским, получаем при прохождении частицы посередине расстояния $D$ между другими частицами вид барьера, показанный на рис. 6, и значение высоты барьера $U_{m}=4 e q D^{-1}$. Отсюда следует зависимость изменения высоты барьера $\varepsilon_{U}=\Delta U_{m} / U_{m}$ от изменения расстояния между „ограничивающими“ частицами $\varepsilon_{D}=\Delta D / D$

$$
\varepsilon_{U}=-\varepsilon_{D}
$$

Из (6) следует, что расчетное относительное изменение высоты барьера с обратным знаком равно относительному изменению „ширины прохода“ одной частицы между двумя „соседними“: при увеличении „ширины прохода“" высота барьера уменьшается. Понимая всю приближенность модели, полученное модельное расчетное соотношение (6) привлечем для сравнения с получаемой экспериментально зависимостью изменения высоты барьера выхода электрона из ловушки от изменения ширины межмолекулярной ловушки в нагружаемом полимере (5).

Можно видеть, что экспериментальное (5) и расчетное (6) соотношения оказались весьма близкими.

Такое совпадение (столь высокая степень которого также, конечно, случайна) может служить некоторым обоснованием того, что полученное экспериментально соотношение (5) правильно выражает причину регистрируемого уменьшения высоты барьера выхода электрона из межмолекулярной ловушки при растягивающем нагружении полимера: увеличение ширины ловушки.

Поэтому можно считать подтвержденным предложенный механизм влияния механического нагружения на электрическую прочность полимеров.

\section{Список литературы}

[1] Физические величины. Справочник / Под ред. И.С. Григорьева, Е.3. Мейлихова. Энергоатомиздат, М. (1991). $1232 \mathrm{c}$.

[2] Т.М. Велиев. Автореф. канд. дис. Институт физики НАН Азербайджана, Баку (1986). 18 с.

[3] S. Nishijima, M. Hara. Cryogenics. 38, 8, 1105 (1998).

[4] Энциклопедия полимеров. Сов. энциклопедия, М. (1977). T. 3. С. $168,1003$.

[5] А.И. Слуцкер, В.Л. Гиляров, Д.Д. Каров, Ю.И. Поликарпов. ФТТ 53, 7, 1255 (2011).

[6] А.И. Слуцкер, Т.М. Велиев, И.К. Алиева, В.А. Алекперов, Ю.И. Поликарпов, Д.Д. Каров. ФТТ 58, 9, 1826 (2016).

[7] М.В. Волькенштейн. Конфигурационная статистика полимерных цепей. Изд-во АН СССР, М.-Л. (1959). 468 с.

[8] Р. Партридж. Радиационная химия макромолекул. Атомиздат, М. (1978). 292 с.

[9] В.А. Закревский, В.А. Пахотин. Высокомолекуляр. соединения. А 23, 3, 658 (1981).

[10] В.А. Марихин, Л.П. Мясникова. Надмолекулярная структура полимеров. Химия, Л. (1977). 240 с.

[11] Л.А. Лайус, А.И. Слуцкер, И.В. Гофман, В.Л. Гиляров, Ю.И. Поликарпов. Высокомолекуляр. соединения А 47, 8, 1394 (2005).

[12] P.C. Arnett. J. Appl. Phys. 46, 12, 5236 (1975). 